\title{
Incidence of Hepatitis B Surface Antigen in Antenatal Cases in a Tertiary Care Hospital in Hyderabad Telangana State, India
}

\author{
Mohammed Ismail Khan ${ }^{1}$, R. Shyamala ${ }^{2}$, Vibha Rani ${ }^{3}$ and K.S. Saraswathi ${ }^{1}$ \\ ${ }^{1}$ Department of Obstetrics and Gynaecology, Shadan Institute of Medical Sciences, India \\ ${ }^{2}$ Department of Microbiology, Malla Reddy Medical College for women, India \\ ${ }^{3}$ Department of Pharmacology, Malla Reddy Medical College for women, India
}

*Corresponding author

\section{A B S T R A C T}

Keywords

HbsAg, Antenatal women, HBV, Viral hepatitis.

Article Info Accepted: 23 December 2016 Available Online: 10 January 2017
This study was done at SIMS and PG Research Centre, Hyderabad. The aim of the study is to find out the incidence of Hepatitis B surface antigen in antenatal women of SIMS during the period of 23 months from January $1^{\text {st }} 2015$ to November 2016. A total of 7096 antenatal women were tested for HBs Ag. out of these 57 pregnant women were found to be positive for $\mathrm{Hbs} \mathrm{Ag}(0.79 \%)$. In pregnancy, Hepatitis B infection with complications to the pregnant women and has the risk of transmission to the baby; which makes it mandatory to screen all antenatal women and their pregnancy should be managed properly and for the newborn immunoprophylaxis is to be given.

\section{Introduction}

Hepatitis B is an acute systemic infection with major pathology in liver, caused by Hepatitis B virus (HBV). More than a third of world's population is estimated to have infected by (HBV). More than a third of world's population is estimated to have been infected by HBV. About $25 \%$ of them become HBV carriers. A quarter of these develop serious liver disease, including chronic hepatitis

Cirrhosis

And Primary hepatic cancer
As there is effective vaccine against HBV, hepatocellular carcinoma has become the only human cancer which is vaccine preventable. The WHO estimates that HBV infection causes more than a million deaths worldwide.

In 1965, Blumberg observed a new antigen in the serum of an Australian aborigine, which was named the Australian antigen. In 1968 it was found to be associated with serum hepatitis, and shown to be the surface component of $\mathrm{HBV}$, hence the name Australia antigen was changed to hepatitis B 
surface antigen (HBsAg). The incubation period is about 1 to 6 months. The infective material is contaminated blood, saliva, vaginal secretions and semen. Vertical transmission to the newborn is about $60-90$ percent.

The maternal complications include abortions, preterm birth, postpartum hemorrhage, infections, hepatic encephalopathy, coagulopathy and renal failure - leading to increased maternal morbidity and mortality.

Acute liver failure occurs in approximately $1 \%$ of patients with acute hepatitis $\mathrm{B}$ and jaundice (Berk et al.,1978). Patients with acute liver failure due to hepatitis B require careful management and monitoring and should be referred rapidly to a tertiary medical centre with the availability of liver transplantation (Hoofnagle et al., 1995).

Perinatal morbidity includes prematurity, intrauterine death, congenital malformations. MTP doesnot alter the prognosis. Universal precautions are taken while conducting the delivery of the HbsAg positive woman. The serum sickness -like syndrome occurs in the setting of acute hepatitis B, often preceding the onset of Jaundice (Alpert et al., 1971).

The serological marker for the diagnosis of acute Hepatitis B infection is the surface antigen $\mathrm{HBs} \mathrm{Ag}$. It appears in patients serum usually 2 to 10 weeks after infection before liver enzymes are increased and clinical symptoms appear. In chronic hepatits, the HBsAg remains elevated for more than 6 months (Yadegari et al., 1998).

\section{Materials and Methods}

This study was conducted at SIMS \& PG
Research Centre Hyderabad from January 1st 2015 to November 2016. In the antenatal clinic, after excluding history of jaundice or previous liver disease, pregnancy induced hypertension and diabetes mellitus, informed consent was taken. They were screened for HbsAg infection by Rapid Immunochromatographic technique. Those found to be positive were confirmed by ELISA test.

\section{Results and Discussion}

Over a duration of 17 months from January 2015 to November 2016, 7096 pregnant women were tested for $\mathrm{HbsAg}, 57$ were found to be positive for HbsAg accounting for an incidence $0.79 \%$. These antenatal cases were in the age group of 16 years to 35 years. 45 were in the age group of 16 years to 25 years accounting for $78.9 \% .12$ were in the age group of 26-35 years.

Hepatitis B infection during pregnancy causes an increase in maternal morbidity and mortality and perinatal mortality in about 20 to $70 \%$. In the present study of 7096 pregnant women 57 were found to be positive for HbsAg accounting for $0.79 \%$

The incidence of Hepatitis B surface antigen varies in different parts of the world and even in India the incidence varies in different states. The antenatal positivity is $6.67 \%$ in Nigeria (Olokoba et al.,2011).

And in Mexico it was $1.67 \%$ (Prakash et al.,1998). In India the antenatal incidence was reported as $4.6 \%$ by Chatterjee et al., at Bangalore (Chatterjee et al.,2009), in Kerala South India it is $0.21 \%$. In a study by Chatterjee et al., the prevalence varied from $0.4 \%$ to $4.6 \%$ with overall mean prevalence of 1.09\% (Ravishankar et al., 2009). 


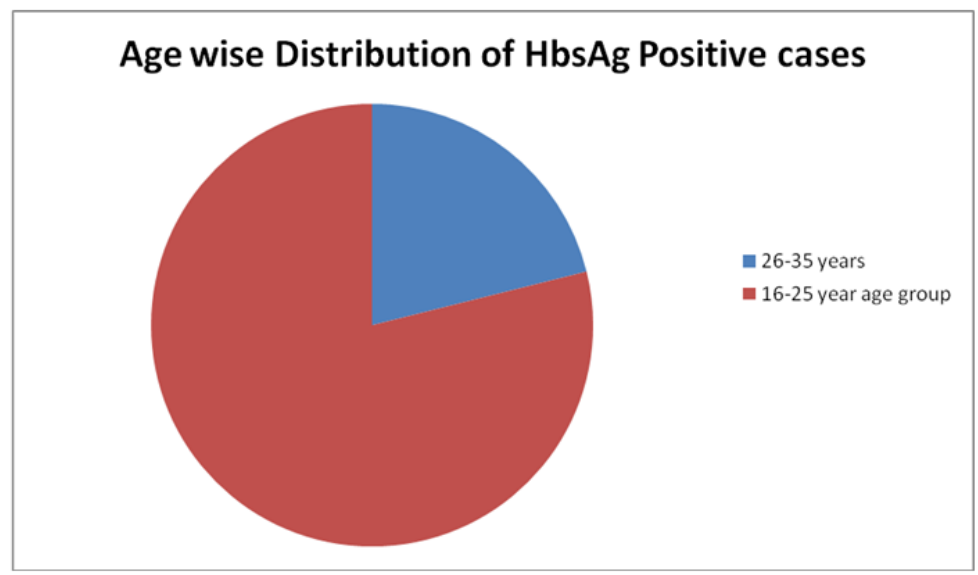

In conclusion, the transmission from mother to child of HbsAg infection during and after delivery is about $90 \%$. In view of the various complications to the mother - leading to increased morbidity and mortality and foetal complication; globally hassled to the development of prophylaxis protocols to decrease the pool of chronic carriers (Asgari $F$ etal). It is mandatory that every pregnant woman is screened, proper interventions are done and immunoprophylaxis of the newborn is done.

\section{References}

Olokoba, A.B. et al. 2011. Nigerian J. clin. Practice, 2011,14.1 10-3,

Alpert, E., et al. 1971. The pathogenesis of arthritis associated with viral hepatitis. Complement -component studies. $N$. Engl. J. Med., 285: 185-189
Asgari, F., Hagazali, M., et al. 2007. Country guide of Hepatitis B care Affairs, Tehran: Ministry of Health.

Berk, P.D., Popper, H. 1978. Fulminant hepatic failure. Am. J. Gastroenterol., 69: 349-400.

Prakash, C. 1998. S.E Asian J. Trop. Med. Public Health, 29: 180-4.

Yadegari, D., S.H. Doaci. 1998. J. Zanjan Med. Sci. Univ. Health Services, 25: 6471.

Hoofnagle, J.H., et al. 1995. Fulminant hepatic failure: summary of a workshop. Hepatol., 21: 240-252.

Ravishanker, K., S. Chatterjee, et al. 2009. Indian Pediatrics, 46: 1008-9.

Chatterjee, S., et al. 2009. Indian Pediatrics, 46: 1005-7.

\section{How to cite this article:}

Mohammed Ismail Khan, R. Shyamala, Vibha Rani, K.S. Saraswathi. 2017. Incidence of Hepatitis B Surface Antigen in Antenatal Cases in a Tertiary Care Hospital in Hyderabad Telangana State. Int.J.Curr.Microbiol.App.Sci. 6(1): 536-538. doi: http://dx.doi.org/10.20546/ijcmas.2017.601.065 